Article

\title{
Transmission Strategies Used by Gyrodactylus gasterostei (Monogenea) on Its Host, the Three-Spined Stickleback Gasterosteus aculeatus
}

\author{
Mayra I. Grano-Maldonado ${ }^{1,2, *}{ }^{(\mathbb{D}}$, Juan Moreno-Navas ${ }^{3}$ and Maria Amparo Rodriguez-Santiago ${ }^{4}$ \\ 1 Departamento de Ciencias Biológicas, Unidad Regional Mazatlán, Universidad Autónoma de Occidente, \\ Av. del Mar 1200, Flamingos, 82149 Mazatlan, Sinaloa, Mexico \\ 2 Facultad de Ciencias Marinas, Universidad Autónoma de Sinaloa, Paseo Claussen s/n. A. P. 610, \\ 82000 Mazatlan, Sinaloa, Mexico \\ 3 Grupo de Oceanografía Física, Universidad de Málaga, 29016 Málaga, Spain; juralon5@hotmail.com \\ 4 CONACyT-Universidad Autónoma del Carmen, Facultad de Ciencias Naturales, Centro de Investigación \\ de Ciencias Ambientales (CICA), Av. Laguna de Términos s/n Col. Renovación 2da Sección, \\ 24155 Ciudad del Carmen, Campeche, Mexico; amparoshalom@hotmail.com \\ * Correspondence: grano_mayra@hotmail.com or mayra.grano@udo.mx; Tel.: +52 6699867520 (ext. 5562)
}

Received: 27 February 2018; Accepted: 23 May 2018; Published: 29 May 2018

\begin{abstract}
The monogenean Gyrodactylus gasterostei and its host, the three-spined stickleback Gasterosteus aculeatus, were employed as a model system to investigate the influence of gyrodactylid maturity on host transfer. Laboratory experiments included the determination of maturity (presence of a male copulatory organ) and reproductive status (presence of a daughter parasite) of the transmitting worms. Parasites with a male copulatory organ were more likely to abandon the host and attempt a host transfer. Additionally, in vivo video observation suggested that detached worms employ water turbulence to assist their effective transfer onto a new host.
\end{abstract}

Keywords: Gyrodactylus; transmission; Monogenea; Gasterosteus aculeatus; maturity; fish

\section{Introduction}

Gyrodactylids are fish ectoparasites (Monogenea). The life-cycle of monogeneans is direct, i.e., transmission does not require an intermediate host. Harris [1] described the mode of reproduction as exceptional; the key feature of this genus is the viviparous mode of reproduction, giving birth to full-sized living individuals, thus allowing fast population growth on the fish host. Infection of new hosts can occur through direct transmission, i.e., occurring when an infected host makes direct skin-to-skin contact with another fish, e.g., fin touching. Four basic transmission profiles were described by Bakke et al. [2]: (1) by contact with live hosts; (2) by contact with dead hosts; (3) by detached parasites drifting in the water column; and (4) by parasites attached to a solid substrate. One of the first descriptions of a specific migratory behavior that facilitates transmission from dead hosts was detailed previously [3-5]. Another remarkable case of gyrodactylid transmission behavior was reported by El-Naggar et al. [6]. These authors indicated that the Gyrodactylus rysavyi Ergens, 1973 demonstrated an exceptionally efficient infection mechanism: this specific parasite was capable of directional swimming by flexing its body, involving $\sim-8$ looping contractions/s with a speed of $1.7-5 \mathrm{~mm} / \mathrm{s}$, and a distance of $\sim 15 \mathrm{~cm}$, allowing to reach its Nile catfish Clarias gariepinus (Burchell) host. However, these authors reported that Macrogyrodactylus congolensis (Prudhoe, 1957) and Macrogyrodactylus clarii (Gusev, 1961) do not possess the ability to perform-twitching or swimming movements, even though they also parasitize the Nile catfish. 
Little is known regarding the biological basis of gyrodactylid host selection and the factors underlying transmission. Regarding this, Dmitrieva's work on Gyrodactylus sphinx and its fish host the Black Sea blenny, Blennius sphinx [7], Valenciennes suggested that the attainment of sexual maturity may trigger the G. sphinx to begin migrating away from host in search of a new one.

Behavioral plasticity in most monogeneans may be necessary in the transmission route to a new host. Bakke et al. [8] described the female and male reproductive system of gyrodactylids. The development of the second daughter occurs by parthenogenesis. In the next generation, development may be by sexual reproduction (after the appearance of the male copulatory organ, MCO) or parthenogenetically. Recent studies have demonstrated that parasites with MCO were willing to transmit or leave the host in different scenarios, such as the use of an anesthetic $[9,10]$.

Parasites that had given birth at least once and developed a MCO were more successful in transmission. In addition, transmission from dead hosts seems to be more resourceful than from living ones [2]. Remaining with a dead host may be a specialized behavior, due to the high risk of transmission in running water and to the increased likelihood of contacting a new host during carcass feeding [11]. The three-spined stickleback is a benthic species; transmission via the substrate may be the most common route. Since the work of Harris et al. [12], there have been other studies on the transmission and population dynamics of Gyrodactylus gasterostei Raeymaekers et al. [13]. Recently, Grano-Maldonado et al. [14-16] have employed this model to study the behavioral basis of host selection.

Although the major routes of Gyrodactylus species transmission have been extensively studied, relatively few studies have examined the behavior of individual gyrodactylids [17] in the transmission process. Specifically, the factors underlying the abandonment of a suitable host are unknown. Therefore, the aim of this study was to investigate the influence of this monogenean maturity on host transfer, using the Gyrodactylus gasterostei / Gasterosteus aculeatus model.

\section{Results}

\subsection{Experiment 1. Parasite Migration from Dead Hosts}

A total of 213 (46.9\%) worms looped off dead hosts, with 88.7\% (186) of those migrating within the first $70 \mathrm{~min}$ following the death of the hosts. Of the migrating worms, $39.6 \%$ gave birth, accounting for $95.2 \%$ of all births observed during the experiment. The majority of migrating worms were mature, i.e., $151(70.9 \%)$ had a male copulatory organ, and 137 (64.6\%) had a daughter in utero. Table 1).

Table 1. Maturity and reproductive status statistical results (Experiment 1).

\begin{tabular}{cccc}
\hline PRS & [A] $n=240$ & {$[\mathrm{~B}] \boldsymbol{n = 2 1 3}$} & $\boldsymbol{p}$-Value (A vs. B) \\
\hline N MCO/ND & $36(15.0 \%)$ & $9(4.2 \%)$ & $<0.001^{* * *}$ \\
N MCO/D & $136(56.7 \%)$ & $53(24.9 \%)$ & $<0.001^{* * *}$ \\
MCO/ND & $25(10.4 \%)$ & $67(31.5 \%)$ & $<0.001^{* * *}$ \\
MCO/D & $43(17.92 \%)$ & $84(39.4 \%)$ & $<0.001^{* * *}$ \\
Total N MCO & $172(71.7 \%)$ & $62(29.1 \%)$ & $<0.001^{* * *}$ \\
Total MCO & $68(28.3 \%)$ & $151(70.9 \%)$ & $<0.001^{* * *}$ \\
\hline
\end{tabular}

*** Significant $p$-value $(p<0.001)$; N, no presence; D, daughter; MCO, male copulatory organ; PRS, parasite reproductive status; $[\mathrm{A}]$, non-migrating parasites; [B], migrating parasites.

\subsection{Experiment 2. Parasite Choice of Live or Dead Hosts}

A total of 1316 parasites were studied, and the experiment was repeated 20 times. The majority contained a daughter in utero but no male copulatory organ (Table 2). The parasites preferred to migrate to live fish instead of dead fish $(p<0.0001)$. Twenty-three $(1.7 \%)$ parasites migrated to the uninfected live fish, 14 (1.1\%) parasites migrated to the uninfected dead fish, $70(5.3 \%)$ parasites looped off but failed to re-attach, and 1209 (91.9\%) parasites remained on the original hosts. 
Table 2. Developmental status of gyrodactylids transferring to either a dead or live host, or dislodged worms collected in the mesh (Experiment 2$)(n=20)$.

\begin{tabular}{cccccc}
\hline \multirow{2}{*}{ Location } & \multicolumn{5}{c}{ Maturity Status of the Gyrodactylid } \\
\cline { 2 - 6 } & N MCO/ND & N MCO/D & MCO/ND & MCO/D & Total \\
\hline Dead-un & $4(4.2 \%)$ & $1(0.2 \%)$ & $8(2.1 \%)$ & $1(0.3 \%)$ & $14(1.1 \%)^{* * *}$ \\
Live-un & $1(1.0 \%)$ & $6(1.1 \%)$ & $11(2.9 \%)$ & $5(1.7 \%)$ & $23(1.7 \%))^{* * *}$ \\
Live-inf & $79(82.3 \%)$ & $510(93.4 \%)$ & $346(90.6 \%)$ & $274(93.8 \%)$ & $1209(91.9 \%)$ \\
Dislodged & $12(12.5 \%)$ & $29(5.3 \%)$ & $17(4.5 \%)$ & $12(4.1 \%)$ & $70(5.3 \%)$ \\
Total & 96 & 546 & 382 & 292 & 1316
\end{tabular}

*** Chi square test $p<0.0001$. Percentage of parasites transmitted in parenthesis. N, no; D, daughter; MCO, male copulatory organ. Live-un, live uninfected; Live-inf, live infected; Dead-un, dead uninfected.

\subsection{Experiment 3. One-to-One Fish Cohabitation}

Of the 3877 parasites collected, $290(7.48 \%)$ migrated from the infected live fish to the uninfected dead fish (Table 3). Of these parasites, $104(35.9 \%)$ were mature and contained a daughter in utero. The Kruskal-Wallis test indicated that there were significant differences $(K=18.66, p<0.001)$ between the parasites remaining on the infected live fish, those that had migrated to the uninfected live fish, those that had migrated to the uninfected dead fish, and those on the control fish (Table 3). The Wilcoxon test indicated that a higher proportion of worms migrated to the dead fish than to the live fish $(W=100 ; p=0.007)$.

\subsection{Experiment 4. Video Monitoring of Gyrodactylid Transmission In Vivo}

The videos clearly showed that the parasites detached from the platform only when the fish swam nearby. In addition, the movement of the parasite matched the direction of the vortex generated by the fish's fin, suggesting that parasite detachment is mediated by water turbulence only. The movement of the parasite through the water column suggests that G. gasterostei exploits the upward trajectory of water vortices generated by the stickleback's fins to assist in transmission (Supplementary Materials Video S1-S4). 
Table 3. Three hours cohabitation experiment on parasite transmission showing the developmental status of gyrodactylids using combinations of fish (Experiment 3) $(n=20)$.

\begin{tabular}{cccccccccccc}
\hline Location & MCO/D & $\%$ & NMCO/D & $\%$ & MCO/ND & $\%$ & NMCO/ND & $\%$ & Total DS & Total & $\%$ \\
\hline Live-un & 46 & $33.3 \%$ & 21 & $15.21 \%$ & 49 & $35.50 \%$ & 22 & $15.94 \%$ & $138(10.49 \%)$ & 1315 & 10.49 \\
Live-inf & 291 & $27.6 \%$ & 447 & $42.36 \%$ & 184 & $17.44 \%$ & 133 & $12.60 \%$ & $1055(80.22 \%)$ & 80.22 \\
Dislodged & 18 & $14.8 \%$ & 13 & $10.65 \%$ & 66 & $54.09 \%$ & 25 & $20.49 \%$ & $122(9.2 \%)$ & 9.27 \\
\hline Dead-un & 104 & $35.86 \%$ & 56 & $19.3 \%$ & 85 & $29.31 \%$ & 45 & $15.51 \%$ & $290(21.96 \%)$ & 1320 & 21.96 \\
Live-inf & 283 & $30.49 \%$ & 399 & $42.99 \%$ & 162 & $17.45 \%$ & 84 & $9.05 \%$ & $928(70.30 \%)$ & 70.30 \\
Dislodged & 25 & $24.50 \%$ & 9 & $8.82 \%$ & 53 & $51.96 \%$ & 15 & $14.70 \%$ & $102(7.72 \%)$ & 7.72 \\
\hline Control & 314 & $27.86 \%$ & 430 & $38.15 \%$ & 243 & $21.56 \%$ & 140 & $12.42 \%$ & $1127(90.74 \%)$ & 1242 & 90.74 \\
Dislodged & 28 & $24.34 \%$ & 25 & $21.73 \%$ & 47 & $40.86 \%$ & 15 & $13.04 \%$ & $115(9.2 \%)$ & 3877 & 3877 \\
Total & 1109 & & 1400 & & 889 & & 479 & & & \\
\hline Average & 7.701 & & 9.72 & & 6.17 & & 3.32 & & & \\
SD & 11.136 & & 17.54 & & 8.00 & & 5.70 & & & \\
\% & 28.60 & & 36.11 & & 22.93 & & 12.35 & & & \\
\hline
\end{tabular}

Live-un, live uninfected; Live-inf, live infected; Dead-un, dead uninfected; Dislodged, dislodged worms collected in the mesh; N, no presence; D, daughter; MCO, male copulatory organ; DS, developmental stages; PS, parasites in the system. 


\section{Discussion}

This study describes several factors, including maturity and reproductive status, that influence the transmission of the parasitic worm G. gasterostei. In Experiment 1, we found that worms with a developed MCO left the host (Table 1). Our results suggest that gyrodactylids that have given birth at least once are more likely to leave the host following its death than are worms that have not yet given birth, according to Harris [12]. Grano-Maldoando [18] found that parasites containing an MCO were significantly more likely to transfer to new hosts than were worms lacking an MCO. The process of leaving a host may also depend on the nutritional status of the worm; perhaps the process requires a filled gut or high energy reserves. [18] observed that lipid droplets were particularly associated with the gut lining, and that there is significant variability in the volume of stored lipid carried by each individual. In this regard, it is possible that the large size of gyrodactylid embryos in utero physically constrains feeding during the latter stages of development by blocking food entry into the intestinal crura. This possibility suggests that older worms have less-filled guts, and hence, would leave less often. Cable et al. [5] reported that detached, starved parasites can abort their embryos and might have a significant influence on the reproductive rate. Previous findings suggest that gyrodactylids give birth to less than four daughters in their lifetime, e.g., Gyrodactylus alexanderi on three-spined sticklebacks [19] and Gyrodactylus bullatarudis on guppies [20].

In Experiment 2 we similarly observed that parasites with an MCO were more likely to leave live hosts than were worms lacking an MCO. This experiment was performed using three different types of hosts in the same chamber (i.e., live infected, live uninfected and dead uninfected), which is less controlled than paired experiments (i.e., using only two types of host at a time). In Experiment 3, which was performed 20 times, we put only one live infected fish, one live uninfected fish, and one dead uninfected fish into the chamber. The dead fish was clearly favorable for the transmission of gyrodactylids (the worms that left the infected fish chose the dead fish over the live one). This result is not surprising, since gyrodactylids that abandon or dislodge from live fish into the water column eventually descend by gravity to the seabed or vessel floor, where dead fish are located. There are no reports indicating that G. gasterostei is capable of directional swimming to reach live fish. Although we did not observe directional swimming in our study, we did not specifically evaluate it, and further studies on this subject are warranted.

El-Naggar et al. [6] reported that G. rysavyi swims to increase its chances of transmission to a new host. Schelkle et al. [21] suggested a swimming-like behavior for the gyrodactylid Ieredactylus rivuli, which infects Rivulus hartii Boulenger in Trinidad.

The three-spined stickleback has been comprehensively studied. This fish is generally considered to be a benthic feeder [22,23]. Hence, the sinking of recently-detached G. gasterostei worms, and their subsequent attachment to dead fish on the seabed or vessel floor, likely increases the parasites' chances of transmitting to a new stickleback. Olstad et al. [11] indicated that the infection of dead fish may also be favorable for gyrodactylid survival: Gyrodactylus salaris worms that remained on a dead salmon host at $18{ }^{\circ} \mathrm{C}$ survived for up to $72 \mathrm{~h}$, whereas worms moving off the dead host survived only for up to $27 \mathrm{~h}$. The authors also indicated that parasites on a dead host live for twice as long as individuals maintained in vitro, probably as a result of sustained feeding. Recent research has demonstrated that G. gasterostei has transmission strategies to reach a new host: the presence of the ultrastructure of coiled musculature myosin and actin fibres component. Also, these parasites are able to store lipids in the gut lining and survive off the host, the three-spined stickleback $[18,24]$.

These interesting phenomena were observed in a period of $1.5-3 \mathrm{~h}$ after starting the experiment, and suggested that well-fed, mature parasites are the most suitable for transmission. Future studies should include a time-course of parasite transmission (i.e., recover transmitted parasites every 5,8 , or $12 \mathrm{~h}$ ), perhaps even evaluating shorter periods (i.e., every 20 or $30 \mathrm{~min}$ ), to comprehensively determine transmission rates in dead and live sticklebacks. Observation could be achieved following a single parasite on the fish using a few drops of neutral red $(0.1 \%)$. 
In our study, no other gyrodactylid species (i.e., Gyrodactylus arcuatus) were found on the surface of the fish. All of the parasites lacked excretory bladders and exhibited a characteristic shape in the ventral bar $[8,25,26])$. There are some features which help to identify grydocatylids, i.e., the MCO (penis) is a spherical and muscular organ with a series of spines which vary in size and number, and are important for the taxonomic differentiation of species. However, the penis is not always present. The shape of the skeletal elements of the opisthaptor, the marginal hooks, the hamuli, and the dorsal and ventral bars are important in the taxonomic identification of the species. Two accessory bars, the ventral and dorsal bars, function to stabilize and coordinate the movements of the hamuli [25,27-29]. In this study, parasites could be compared with other photographs of Gyrodactylus [29,30].

In our study, no other gyrodactylid species (i.e., G. arcuatus) were found on the surface of the fish. All of the parasites studied lacked excretory bladders, and had ventral bar with short processes characteristic for G. gasterostei. This observation may explain why the sticklebacks differed in the natural infection levels of $G$. arcuatus [26,31,32].

A recent study elaborated by Lumme et al. [33] in Baltic populations of the three-spined stickleback G. aculeatus (L.) demonstrated that the transmission between host generations may present a model for studying the population dynamics of the parasite, which is important for understanding the evolution and coevolution of the host-parasite system. The local mitochondrial diversity of Gyrodactylus arcuatus (Bychowsky, 1933), is very high while the spatial differentiation is weak. For this reason, transmission can be studied at the level of haplotypes. The main results derived from this research are that terms such as 'nesting and polygamy' are suggested as factors maintaining the high genetic diversity of the parasite population. Also, the transmission dynamics and the population structure of G. arcuatus (Baltic strain) is fundamentally different from that of G. salaris (Malmberg, 1957), parasitizing the Baltic salmon Salmo salar (L.), which is a very important conclusion, given the impact that G. salaris has had on Atlantic Salmon strains [8].

Using video recording, we observed that worms may employ water turbulence to detach from a substrate and move towards the fish. The slow-motion videos clearly showed that the parasites detached exactly when the fish swam nearby, not before or after, and that the movement of the parasites after detachment matched the direction of the vortex generated by the passing fish's fins. No free parasites were observed in the absence of nearby fish, indicating that parasite detachment was due solely attributable to the water turbulence created by the fish. It is possible that parasites may initially detect water movements associated with an approaching host using mechano-chemical and photosensitive receptors [15]. We observed that complex water flows, e.g., the pectoral-fin wake, may improve parasite transmission (see, for example, supplementary video S3). The effects of water turbulence have been studied by a number of investigators [34-36], in order to examine the locomotion of the rainbow trout Oncorhynchus mykiss. It was found that the pectoral fins produced circular vortices capable of transporting several particles (i.e., parasites) onto the fish's body. Perhaps these vortices could be used by G. gasterostei in the same way. We propose that water turbulence may comprise an additional gyrodactylid transmission route, although its relative importance with respect to the other recognized routes is unknown. More evidence, and an effective particle-tracking experiment detailing the hydrodynamic forces, are clearly needed.

Fish skin and gills may undergo morphological and/or histopathological changes when exposed to substances which may damage tissues or influence gyrodactylid transmission. Anesthetic, for example, was shown not to have an effect on gyrodactylid transmission [10]. However, formaldehyde was found to cause mild hyperplasia and detachment of respiratory epithelium in the gills of juvenile Mugil liza (Valenciennes) [37]. Fish tolerance to formaldehyde may be species-specific. Unfortunately, in our study, we did not examine the probable effect of formaldehyde on fish. To the best of our knowledge, all fish used in our experiments did not exhibit any erratic behavior or skin disorder. Future studies are warranted to evaluate whether the effects of formaldehyde, including possible gill and skin damage, affect gyrodactylid transmission in sticklebacks. 
The possibility of finding just one species of Gyrodactylus did not raise suspicion; some authors $[13,26,31,32,38]$ have investigated local adaptation and maturity, showing that in the Gasterosteus-Gyrodactylus example, differential host immune resistance may instead explain differential accumulation of parasites. To explain why local adaptation appears lacking, we must understand the strength of selection and the population genetic structure of both parasite and host. To understand why immune resistance is different among populations, we must (1) rule out adaptation to the laboratory with extra replication of populations incorporated in a full factorial design; (2) test for possible trade-offs or genetic linkage with other important fitness-related traits; and (3) rule out the effects of drift and inbreeding. Finally, to understand why immune resistance changes with life stage, we need to test infection rates in more natural settings, such as by reciprocal transplant experiments.

Our results indicate that personnel involved in fish disease activities, e.g. aquaculture, should remove dead fish as soon as possible, in order to minimize parasite transmission in the bottom of the tank. Personnel should also periodically check for the presence of parasites in order to accurately assess the risk of parasite transmission.

\section{Materials and Methods}

Four experiments were conducted to assess parasite behavior: migration from dead hosts; choice of live or dead hosts; one-to-one fish cohabitation; and video monitoring of gyrodactylid transmission in vivo.

\subsection{Source of Hosts and Parasites}

An experimental model using G. gasterostei and its host, the stickleback G. aculeatus, was used in this study. The naturally-infected fish were collected from a settlement pond which feeds a commercial fish farm on a branch of the River Allan near Stirling, Stirlingshire, Scotland (56.1104917, -3.97368). The fish were transferred to an aquarium facility at the Institute of Aquaculture, University of Stirling, where they were held in 25-L black plastic tanks containing aerated "home" stream water at $15 \pm 1{ }^{\circ} \mathrm{C}$. The fish were fed ad libitum on a diet of frozen bloodworms (Gamma, Chorleywood, UK). Uneaten food and solid wastes were removed daily using a siphon, after which the water (dechlorinated using an ELGA C960 carbon filter (ELGA\&VEOLIA LAB, High Wycombe, UK), aerated, and conditioned at $15 \pm 1{ }^{\circ} \mathrm{C}$ ) was replaced. Dead fish were removed from the tank. Dissolved oxygen levels were nearly $100 \%$, salinity was $5 \mathrm{~g} / \mathrm{L}$, and light conditions (measured with a handheld light meter) were 2800 lux on a 12-h photoperiod. The fish were given a minimum of $48 \mathrm{~h}$ following capture to adjust to the tanks before experimentation. Feeding was stopped one day prior to each experiment, in order to maintain water quality and reduce fish stress. All experiments were performed in accordance with a Home Office UK License (Act 1986 Scientific Procedures on Living Animals PPL No. 60/4088, 60/4715).

\subsection{Parasite Free-Hosts}

Parasite-free G. aculeatus were obtained by treating selected fish (with few or no visible parasites) with $300 \mathrm{mg} / \mathrm{L}$ of formaldehyde for $60 \mathrm{~min}$ under constant aeration. Following treatment, the fish were transferred to a recovery tank containing clean, aerated, dechlorinated water. The fish were maintained for 10 days following treatment, and examined again prior to use to ensure that they were parasite-free. If the fish were still infected, they were given a second formalin treatment. Prior to experimentation, the fish were anaesthetized with $0.01 \mathrm{M}$ 2-phenoxyethanol (MERCK, Darmstadt, Germany), and examined under an Olympus SZ30 stereo microscope (Olympus, Tokio, Japan) to ensure that no parasites were present. Afterwards, the fish were maintained for 3 more days before commencing experiments.

\subsection{Experiment 1. Parasite Migration from Dead Hosts}

Twenty individual sticklebacks were euthanized with 2-phenoxyethanol, and placed in individual Petri dishes containing clean water at $15^{\circ} \mathrm{C}$. The fish were observed under an Olympus 
SZ30 stereomicroscope for $100 \mathrm{~min}$, then all of the gyrodactylids that had migrated off the fish to the bottom of the petri dish were collected with a $200 \mu \mathrm{L}$ pipette. Each parasite was transferred to a slide, coverslipped with a drop of Malmberg's fixative (ammonium picrate glycerine), and examined for maturity status using a compound microscope (Olympus BX51, Olympus) with a $100 \times$ oil-immersion objective. The fish carcasses containing non-migrated parasites were fixed in $80 \%$ ethanol, then these parasites were examined for maturity status. The hard parts of the opisthaptor were used to identify the gyrodactylid species. The maturity status was classified into four developmental states (Figure 1): (1) no daughter present in utero and no male copulatory organ (ND/N MCO); (2) no male copulatory organ, but a daughter present (N MCO/D); (3) male copulatory organ present, but no daughter (MCO/ND); and (4) both male copulatory organ and daughter present (MCO/D). A daughter was considered to be present if the rudimentary hard parts of the opisthaptor or attachment hooks were evident. The male copulatory organ was considered to be present only if the spines were clearly visible (Figure 1). A Pearson's Chi-squared test was used to determine whether the presence of a male copulatory organ in the migrating and non-migrating parasites correlated with successful transmission. The anesthetic itself (0.01 M 2-phenoxyethanol) did not affect the transmission of Gyrodactylus in a preliminary study.

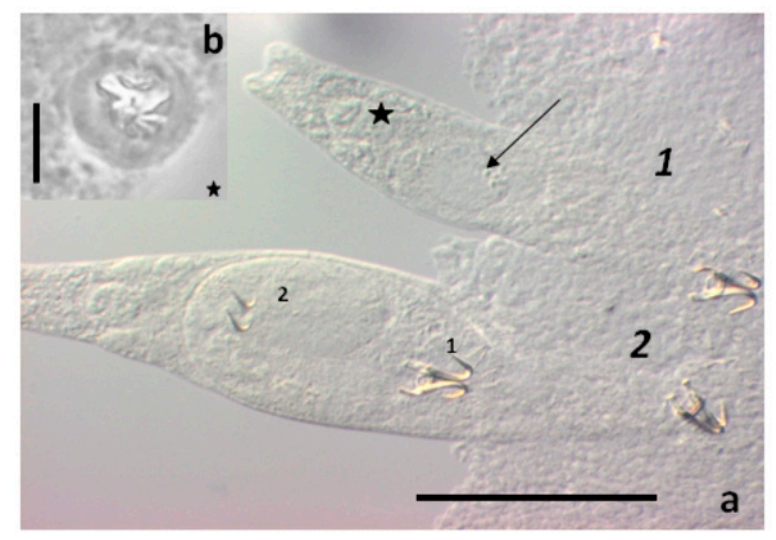

Figure 1. Light micrographs of Gyrodactylus gasterostei. (a) An individual parasite (1) showing an empty uterus (arrow) and pharynx (star), and another parasite (2) showing the presence of a daughter (1), (and granddaughter (2) in utero. Scale bar $=0.5 \mathrm{~mm}$; (b) Male copulatory organ. Scale bar $=10 \mu \mathrm{m}$.

\subsection{Experiment 2. Parasite Choice of Live or Dead Hosts}

Infected sticklebacks were cohabited with one uninfected live and one dead fish in a $200 \mathrm{~mL}$ chamber for $3 \mathrm{~h}$ at $15^{\circ} \mathrm{C}$, under ambient light conditions (2800 lux). After the period of cohabitation, the live fish were euthanized and all were immediately preserved in $80 \%$ ethanol to fix the gyrodactylids without changing the parasite's density infrapopulation. The water from each chamber was also mesh-filtered $(20 \mu \mathrm{m})$ in order to collect the free parasites. Parasite maturity status was determined using the same protocol as in Experiment 1. A Chi-squared test was used to determine whether host status (alive vs. dead) correlated with successful transmission. The experiment was performed 20 times.

\subsection{Experiment 3. One-to-One Fish Cohabitation}

To exclude the possibility that the existence of other fish in the chamber influenced host transfer in Experiment 2, only three fish were used in Experiment 3: one infected live fish was cohabited with one uninfected live fish and one uninfected dead fish. This experiment was also performed 20 times. An uninfected live fish alone in the chamber was used as a control to determine whether parasites attempt to transit without a fish trigger. Parasite maturity status was determined using the same protocol as in Experiment 1. Kruskal-Wallis \& Wilcoxon non-parametric tests were used to evaluate whether host status (alive vs. dead) correlated with successful transmission (Table 3). 


\subsection{Experiment 4. Video Monitoring of Gyrodactylid Transmission In Vivo}

During transmission, parasites may dynamically use water turbulence and vortexes formed by water and fish movements. To investigate this possibility, the movement of parasites during transmission were tracked by video recording, using a $16 \times 15 \times 8.5 \mathrm{~cm}$ experimental chamber with a viewing platform positioned within an $80 \mathrm{~cm}$-square translucent photo tent. A Veho Discovery Deluxe USB microscope (Veho, Southampton Hampshire, UK) with 400× magnification (VMS-004D) was focused on the platform, and relayed the video directly to a computer. The video was edited using Adobe Premiere Pro 1.5 (Adobe, San Jose, CA, USA) for creating the graphics, and Windows Movie Maker 5.1 (Microsoft. Redmond, WA, USA) for editing the original footage. For the study, parasites $(n=9)$ were dislodged from a fish host and placed individually with a micropipette into the middle of the viewing platform. One non-infected fish was introduced into the chamber, and video recording was started. Nine videos were recorded, showing different scenarios (dislodge parasites reaching swimming fish; dislodge parasites employing turbulence and vortex to reach the swimming fish). However, we employed just four in order to show our best results.

Supplementary Materials: The following are available online at http://www.mdpi.com/2410-3888/3/2/ 20/s1. Video S1 and S2: Video footage showing the movement of a detached gyrodactylid from bottom (white circle) moving upwards and attaching to the ventral zone of the three-spined stickleback host (green circle); Video S3 and S4: Video footage showing the movement of a detached gyrodactylid from bottom (white arrow) moving upwards (green arrow) to three-spined stickleback using turbulence generated by the fin's fish as a hydrodynamic force assisting upwards transmission. During the video a clear observation of several vortexes (arrow) produced by the pectoral fin fish motion.

Author Contributions: M.I.G.-M. And J.M.-N. conceived and designed the experiments; M.I.G.-M. performed the experiments; M.A.R.-S. And J.M.-N. analyzed the data; M.A.R.-S. contributed with materials and software for analysis; M.I.G.-M. wrote the paper, three authors read and provide feedback.

Acknowledgments: We thank Andy Shinn and James Bron for mentorship; Ian Bricknell and Rod Wootten (Rest In Peace) for assistance as committee members and editorial comments. This article is derived from M.I.G.-M.'s thesis completed at Stirling University. We thank Iain Semple for help in the collection of fish from Howietown fish farm. This study was supported by a Department of the Environment, Food and Rural Affairs (Defra) and an Overseas Research Students Awards Scheme (ORSAS) UK and Consejo Nacional Ciencia y Tecnología, Mexico CONACyT (research fellow No. 171032).

Conflicts of Interest: The authors declare no conflict of interest.

\section{References}

1. Harris, P.D. Interactions between reproduction and population biology in gyrodactylid monogeneans-A review. Bull. Fr. Peche Piscic. 1993, 1, 47-65. [CrossRef]

2. Bakke, T.A.; Harris, P.D.; Cable, J. Host specificity dynamics: Observations on gyrodactylid monogeneans. Int. J. Parasitol. 2002, 32, 281-308. [CrossRef]

3. Cable, J.; Harris, P.D. Gyrodactylids developmental biology historical review, current status and future trends. Int. J. Parasitol. 2002, 32, 255-280. [CrossRef]

4. Harris, P.D. Changes in the site specificity of Gyrodactylus turnbulli Harris, 1986 (Monogenea) during infections of individual guppies (Poecilia reticulata Peters, 1859). Can. J. Zool. 1988, 66, 2854-2857. [CrossRef]

5. Cable, J.; Scott, E.C.G.; Tinsley, R.C.; Harris, P.D. Behaviour favoring transmission in the viviparous monogenean Gyrodactylus turnbulli. J. Parasitol. 2002, 88, 183-184. [CrossRef]

6. El-Naggar, M.M.; El-Naggar, A.M.; Kearn, G.C. Swimming in Gyrodactylus rysavyi (Monogenea: Gyrodactylidae) from the Nile catfish Clarias gariepinus. Acta Parasitol. 2004, 49, 102-107.

7. Dmitrieva, E.V. Transmission triggers and pathways in Gyrodactylus sphinx (Monogenea, Gyrodactylidae). Vestnik Zool. 2003, 37, 67-72.

8. Bakke, T.A.; Cable, J.; Harris, P.D. The biology of gyrodactylid monogeneans: The "Russian Doll-killers". Adv. Parasitol. 2007, 64, 161-376. [PubMed]

9. Grano-Maldonado, M.I. The Biological and Behavioural Basis of Host Selection in Transmission of Gyrodactylus (Monogenea). Ph.D. Thesis, Stirling Univeristy, Stirling, UK, 2011; p. 228. 
10. Grano-Maldonado, M.I.; Palaiokostas, C. Does the anaesthetic influence behavioural transmission of the monogenean Gyrodactylus gasterostei Gläser, 1974 off the host? Helminthologia 2015, 52, 144-147. [CrossRef]

11. Olstad, K.; Cable, J.; Robertsen, G.; Bakke, T.A. Unpredicted transmission strategy of Gyrodactylus salaris (Monogenea: Gyrodactylidae): Survival and infectivity of parasites on dead hosts. Parasitology 2006, 133, 33-41. [CrossRef] [PubMed]

12. Harris, P.D. Observations on the development of the male reproductive system in Gyrodactylus gasterostei Gläser, 1974 (Monogenea, Gyrodactylidae). Parasitology 1985, 91, 519-529. [CrossRef]

13. Raeymaekers, J.; Wegner, K.; Huyse, T.; Volckaert, F. Infection dynamics of the monogenean parasite Gyrodactylus gasterostei on sympatric and allopatric populations of the three-spined stickleback Gasterosteus aculeatus. Folia Parasitol. 2011, 58, 27-34. [CrossRef] [PubMed]

14. Grano-Maldonado, M.I.; Bron, J.; Longshaw, M.; Shinn, A. The accidental transfer of Gyrodactylus (Monogenea) during short duration fish transportation. Fish Pathol. 2011, 46, 71-79. [CrossRef]

15. Grano-Maldonado, M.I. Ultrastructure of the external sensory apparatus of Gyrodactylus gasterostei Gläser, 1974. Microsc. Res. Tech. 2014, 77, 740-747. [CrossRef] [PubMed]

16. Grano-Maldonado, M.I. Gyrodactylus gasterostei a difficult meal to swallow for the three-spined sticklebacks, Gasterosteus aculeatus L. Scanning 2014, 36, 614-621. [CrossRef] [PubMed]

17. Brooker, A.J.; Grano-Maldonado, M.I.; Irving, S.; Bron, J.; Longshaw, M.; Shinn, A.P. The effect of octopaminergic compounds on the behaviour and transmission of Gyrodactylus. Parasite Vectors 2011, 4, 207. [CrossRef] [PubMed]

18. Grano-Maldonado, M.I. Ultrastructure study of the stored lipid reserves in Gyrodactylus gasterostei Gläser, 1974 using confocal microscopy. J. Microsc. Ultrastruct. 2017. [CrossRef]

19. Lester, R.J.G.; Adams, J.R. Gyrodactylus alexanderi: Reproduction, mortality and effect on its host Gasterosteus aculeatus. Can. J. Zool. 1974, 52, 827-833. [CrossRef] [PubMed]

20. Scott, M. Reproductive potential of Gyrodactylus bullatarudis (Monogenea) on guppies (Poecilia reticulata). Parasitology 1982, 85, 217-236. [CrossRef]

21. Schelkle, B.; Paladini, G.; Shinn, A.; King, S.; Johnson, M.; van Oosterhout, C.; Mohammed, R.; Cable, J. Ieredactylus rivuli gen. et sp. nov. (Monogenea, Gyrodactylidae) from Ivulus hartii (Cyprinodontiformes, Rivulidae) in Trinida. Acta Parasitol. 2011, 56, 360-370. [CrossRef]

22. Lavin, P.; McPhail, J. The evolution of freshwater diversity in the three-spined stickleback (Gasterosteus aculeatus): Site-specific differentation of trophic morphology. Can. J. Zool. 1985, 63, 2632-2638. [CrossRef]

23. Bell, M.; Foster, S. The Evolutionary Biology of the Three spined Stickleback; Oxford University Press: New York, NY, USA, 1994.

24. Grano-Maldonado, M.I.; Bruno de Sousa, C.; Rodriguez-Santiago, A. First insights into the ultrastructure of myosin and actin bands using Transmission Electron Microscopy in Gyrodactylus (Monogenea). J. Microsc. Ultrastruct. 2017. [CrossRef]

25. Malmberg, G. The excretory system and marginal hooks as a basis for the systematics of Gyrodactylus (Trematoda, Monogenea). Arch. Zool. 1970, 23, 1-237.

26. De Roij, J.; Harris, P.; MacColl, A. Divergent resistance to a monogenean flatworm among three-spined stickleback populations. Funct. Ecol. 2011, 25, 211-217. [CrossRef]

27. Harris, P.D.; Shinn, A.P.; Cable, J.; Bakke, T.A. Nominal species of the genus Gyrodactylus von Nordmann 1832 (Monogenea: Gyrodactylidae), with a list of principal host species. Syst. Parasitol. 2004, 59, 1-27. [CrossRef] [PubMed]

28. Shinn, A.P.; Hansen, H.; Olstad, K.; Bachmann, L.; Bakke, T.A. The use of morphometric characters to discriminate species of laboratory-reared and wild populations of Gyrodactylus salaris and G. thymalli (Monogenea). Folia Parasitol. 2004, 51, 239-252. [CrossRef] [PubMed]

29. Harris, P.D.; Shinn, A.P.; Cable, J.; Bakke, T.A.; Bron, J.E. GyroDb: Gyrodactylid monogeneans on the web. Trends Parasitol. 2008, 24, 109-111. [CrossRef] [PubMed]

30. Shinn, A.P.; Harris, P.D.; Cable, J.; Bakke, T.A.; Paladini, G.; Bron, J.E. GyroDb-A Home for Gyrodactylids on the Web. Editors 2010. GyroDb. World Wide Web Electronic publicatIon, Version (06/2010). Available online: www.gyrodb.net (accessed on 1 August 2016).

31. Konijnendijk, N.; Raeymaekers, J.A.; Vandeuren, S.; Jacquemin, L.; Volckaert, F.A. Testing for local adaptation in the Gasterosteus-Gyrodactylus host-parasite system. Evol. Ecol Res. 2013, 15, 489-502. 
32. Neiman, M.; Sharbel, T.F.; Schwander, T. Genetic causes of transitions from sexual reproduction to asexuality in plants and animals. J. Evol. Biol. 2014, 27, 1346-1359. [CrossRef] [PubMed]

33. Lumme, J.; Ziętara, M.S. Horizontal transmission of the ectoparasite Gyrodactylus arcuatus (Monogenea: Gyrodactylidae) to the next generation of the three-spined stickleback Gasterosteus aculeatus. Folia Parasitol. 2018, 65, 006. [CrossRef] [PubMed]

34. Tytell, E.D. Median fin function in bluegill sunfish, Lepomis macrochirus: Streamwise vortex structure during steady swimming. J. Exp. Biol. 2006, 209, 1516-1534. [CrossRef] [PubMed]

35. Bozkurttas, M.; Dong, H.; Mittal, R.; Madden, P.; Lauder, G.V. Hydrodynamic performance of deformable fish fins and flapping foils. In Proceedings of the 44th AIAA Aerospace Sciences Meeting and Exhibit, Reno, NV, USA, 9-12 January 2006; 2006 AIAA Paper 2006-1392.

36. Drucker, E.G.; Lauder, G.V. Function of pectoral fins in rainbow trout: Behavioral repertoire and hydrodynamic forces. J. Exp. Biol. 2003, 206, 813-826. [CrossRef] [PubMed]

37. Pahor-Filho, E.; Miranda Filho, K.C.; Klosterhoff, M.; Romano, L.A.; Pereira Júnior, J. Histopathological and behaviour effects of formaldehyde treatment in juvenile mullet, Mugil liza (Valenciennes). Aquac. Res. 2015, 46, 3040-3045. [CrossRef]

38. Harris, P.D. Ecological and genetic evidence for clonal reproduction in Gyrodactylus gasterostei Gläser, 1974. Int. J. Parasitol. 1998, 28, 1595-1607. [CrossRef]

(C) 2018 by the authors. Licensee MDPI, Basel, Switzerland. This article is an open access article distributed under the terms and conditions of the Creative Commons Attribution (CC BY) license (http:/ / creativecommons.org/licenses/by/4.0/). 